Recepción: 14 / 04 / 2018

Aceptación: 26 / 06 / 2018

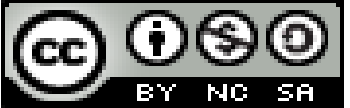

Ciencias de la salud

Publicación: 15 / 09 / 2018

Artículo de investigación

\title{
Prevalencia de la lactancia materna exclusiva en la alimentación complementaria
}

Prevalence of exclusive breastfeeding in complementary feeding

\section{Prevalência de amamentação exclusiva na alimentação complementar}

\author{
Isabel R. Ríos-Saavedra ${ }^{\mathrm{I}}$ \\ isabelrios@gmail.com \\ Melania C. Alcivar-García II \\ melaniaalcivar@gmail.com \\ Monserrat B. Pico-Franco III \\ monserratpico@gmail.com
}

\section{Correspondencia: isabelrios@gmail.com}

I Doctora en Medicina y Cirugía, Magister en Ginecología Infanto Juvenil y Salud Reproductiva del Adolescente, Magister en Gerencia Clínica en Salud Sexual y Reproductiva, Docente de la Universidad Técnica de Manabí, Portoviejo, Ecuador.

II Doctora en Medicina y Cirugía, Magister en Gerencia Educativa, Magister en Gerencia Clínica en Salud Sexual y Reproductiva, Especialista en Pediatría, Docente de la Universidad Técnica de Manabí, Portoviejo, Ecuador.

III Doctora en Medicina y Cirugía, Magister en Gerencia Clínica en Salud Sexual y Reproductiva, Especialista en Pediatría, Docente de la Universidad Técnica de Manabí, Portoviejo, Ecuador. 


\title{
Resumen
}

Actualmente los hábitos alimenticios, durante los primeros años de vida, son conducentes con ciertos grados de desnutrición que abarca desde la poca ganancia ponderal hasta la obesidad en algunos casos en los cuales el paciente se encuentra desnutrido, constituyéndose en un problema de agresivo crecimiento en América Latina, de la cual no escapa el Ecuador, tal es el caso de madres que asisten a la consulta externa con hijos en periodos de lactancia materna exclusiva al hospital Rodríguez Zambrano ubicado en el cantón de Manta de la Provincia de Manabí, Ecuador. El objetivo, se centró en conocer la prevalencia de la lactancia materna exclusiva, durante el inicio de la alimentación complementaria. La metodología fue de tipo descriptiva correlacional y de campo. La población la constituyeron 120 madres que asistieron a la consulta durante el segundo semestre del año 2017. El instrumento utilizado fue un cuestionario estructurado de preguntas cerradas y 3 alternativas de repuestas, y el análisis de la información se hizo a través de la estadística descriptiva inferencial. Entre sus resultados se pudo evidenciar que priva la educación secundaria en el $68 \%$ de las madres consultadas y el $42 \%$ daban alimentación mixta a los seis meses de edad y en el 69\% daban alimentación a través de fórmulas lácteas.

Palabras claves: alimentación mixta; alimentación complementaria; nutrición y lactancia materna.

\begin{abstract}
Currently, eating habits, during the first years of life, are conducive to certain degrees of malnutrition ranging from low weight gain to obesity in some cases in which the patient is malnourished, becoming a problem of aggressive growth in America Latina, from which Ecuador does not escape, such is the case of mothers who attend the outpatient clinic with children during periods of exclusive breastfeeding at the Rodríguez Zambrano hospital located in the Manta canton of the Province of Manabí, Ecuador. The objective, focused on knowing the prevalence of exclusive breastfeeding, during the start of complementary feeding. The methodology was descriptive correlational and field type. The population consisted of 120 mothers who attended the consultation during the second semester of the year 2017. The instrument used was a structured questionnaire of closed questions and 3 alternative answers, and the analysis of the information was made through inferential descriptive statistics. Among its results it was evident
\end{abstract}


that deprived secondary education in $68 \%$ of the mothers consulted and $42 \%$ gave mixed feeding at six months of age and in $69 \%$ gave food through milk formulas.

Keywords: mixed feeding; supplementary feeding; nutrition and breastfeeding.

\section{Resumo}

Atualmente, os hábitos alimentares, durante os primeiros anos de vida, favorecem certos graus de desnutrição, desde baixo ganho de peso à obesidade, em alguns casos em que o paciente está desnutrido, tornando-se um problema de crescimento agressivo na América. Latina, do qual o Equador não escapa, como é o caso das mães que freqüentam o ambulatório com crianças durante os períodos de amamentação exclusiva no hospital Rodríguez Zambrano, localizado no cantão de Manta, província de Manabí, Equador. O objetivo, focado em conhecer a prevalência do aleitamento materno exclusivo, durante o início da alimentação complementar. A metodologia foi descritiva correlacional e tipo de campo. A população foi composta por 120 mães que compareceram à consulta durante o segundo semestre do ano de 2017. O instrumento utilizado foi um questionário estruturado de questões fechadas e três alternativas de respostas, sendo a análise das informações feita por meio de estatística descritiva inferencial. . Entre seus resultados, ficou evidente que o ensino médio carente em $68 \%$ das mães consultadas e $42 \%$ davam alimentação mista aos seis meses de idade e em $69 \%$ davam alimentos por meio de fórmulas lácteas.

Palavras chave: alimentação mista; alimentação complementar; nutrição e amamentação.

\section{Introducción}

La Organización Mundial de la Salud (OMS, 2017) y el Fondo de Naciones Unidas para la Infancia (Unicef, 1989), señalan que la lactancia es una forma inigualable de facilitar el alimento ideal para el crecimiento y desarrollo correcto de los niños, ambas organizaciones recomiendan como imprescindible la lactancia materna exclusiva durante los seis primeros meses del recién nacido. También recomiendan seguir amamantando a partir de los seis meses, al mismo tiempo que se va ofreciendo al bebé otros alimentos propios para su edad complementarios, hasta un mínimo de dos años. Por otro lado, afirman que a partir de los dos primeros años la lactancia 
materna tiene que mantenerse hasta que el niño o la madre decidan, sin que exista ningún límite de tiempo. No se sabe cuál es la duración "normal" de la lactancia materna en la especie humana. Los referentes sobre los términos y características de la lactancia se comprenden desde el contexto cultural de las madres que lactan, de tal forma que los periodos de lactancia se pueden extender tanto como la variabilidad de culturas existentes en el mundo. En el mundo podemos referir casos en donde se considera un acto únicamente de bebés de pocas semanas o meses, así como también se encuentran casos en los que se ha mantenido la lactancia durante varios años. (Wright, 2001).

Sin embargo, la frecuencia y duración de la lactancia materna disminuyeron rápidamente a lo largo del siglo XX. En 1955 se había creado en las Naciones Unidas el Grupo Asesor en Proteínas (GAP), para ayudar a la OMS a ofrecer consejo técnico a Unicef y FAO en sus programas de ayuda nutricional y asesoría sobre la seguridad y la adecuación del consumo humano de nuevos alimentos proteicos. En los años setenta el GAP emite informes donde advierte la preocupación por el problema de la malnutrición infantil derivada del abandono de la lactancia materna e invita a la industria a cambiar sus prácticas de publicidad de productos para la alimentación infantil.

La práctica de lactancia materna durante los primeros meses y años de vida ha demostrado ser un factor importante para prevenir la aparición de enfermedades a corto plazo como las infecciones, hasta aquellas crónicas que se presentan en el transcurso de la vida como la diabetes, obesidad o ciertos tipos de cáncer. Además, ayuda a prevenir la malnutrición, desde la desnutrición crónica, que en Ecuador asciende al 25.3\%, hasta la obesidad y sobrepeso, que llega al 62,8\% en adultos (de 19 a 60 años) según la ENSANUT 2012.

De acuerdo a la información obtenida por la ENSANUT-ECU 2012, se conoce que las prevalencias de lactancia materna exclusiva y continua no alcanzan los niveles adecuados ni metas establecidas por el Plan Nacional del Buen Vivir (PNBV) 2013-2017, la cual establece como meta el aumentar la prevalencia de lactancia materna exclusiva en los primeros 6 meses de vida. Con base a estas exposiciones, se propone en este artículo conocer la prevalencia de la lactancia materna exclusiva, durante el inicio de la alimentación complementaria. 
La lactancia materna, según Torras (2001), es la forma ideal de aportar a los niños pequeños los nutrientes que necesitan para un crecimiento y desarrollo saludables. Prácticamente todas las mujeres pueden amamantar, siempre que dispongan de buena información y del apoyo de su familia y del sistema de atención de salud. Vahlquist (1981), recomienda la lactancia materna exclusiva durante seis meses, la introducción de alimentos apropiados para la edad y seguros a partir de entonces, y el mantenimiento de la lactancia materna hasta los 2 años o más.

Así mismo, la Organización Mundial de la Salud (OMS, 2017) recomienda tomar en cuenta para la lactancia materna:

Iniciar la lactancia materna en la primera hora de vida y mantenerla como único alimento hasta los seis meses de vida, y como complemento hasta los dos años. Es la forma ideal de aportar a los niños pequeños los nutrientes que necesitan para un crecimiento y desarrollo saludable.

La leche materna es el único alimento que necesita el bebé. Contiene todos los nutrientes para favorecer su crecimiento y desarrollo, aumenta las defensas contra las enfermedades y estrecha el vínculo de amor entre la madre y su hijo o hija.

Las mujeres que trabajan o estudian necesitan el apoyo de su pareja, familia y jefes, para continuar alimentando a su bebé con leche materna.

La mujer no es la única responsable de la lactancia materna, es importante saber que el éxito depende del apoyo constante de la pareja y familia.

Acudir a la Unidad de Salud más cercana para recibir consejería y preparación necesarias.

\section{Ventajas de la alimentación materna}

\section{Prevención de infecciones}

Una infección que se previene o reduce su frecuencia gracias a la alimentación con leche materna es la gastroenteritis, la más importante por lo menos en países en vías de desarrollo. Esta enfermedad es muy rara en los lactantes que se alimentan sólo de leche humana. La enterocolitis necrosante, por otra parte, ocurre con menor frecuencia en los alimentados al seno que en los que reciben leche de vaca. Además, la leche materna proporciona protección contra el cólera. Muchos 
estudios señalan que hay una menor incidencia de infección respiratoria en niños alimentados al seno. Otra razón obvia para mayor incidencia de gastroenteritis debido a la alimentación con fórmulas es la contaminación de botellas y tetillas, así como por mal almacenamiento. Por su parte, el botulismo infantil se limita virtualmente a los lactantes alimentados con leche industrializada. El menor riesgo de muerte infantil súbita infantil en niños alimentados al seno no se explica por completo.

\section{Prevención de sensibilizaciones alimentarias}

Está ampliamente demostrada la presencia de alérgenos alimentarios en la leche materna, tales como proteínas de huevo, de gluten (contenidas en el trigo, la cebada, el centeno, la avena, y todos sus híbridos), de leche de vaca y de cacahuete. En general, las concentraciones se relacionan con la cantidad del alimento ingerido por la madre. Los alérgenos pasan con rapidez a la leche materna, en los minutos posteriores a la ingesta, y pueden permanecer durante varias horas. Se ha sugerido que esta exposición a alérgenos alimentarios a través de la lactancia induce la tolerancia en el bebé, si bien actualmente no se ha establecido cómo y cuándo exponer a los bebés a los alérgenos alimentarios potenciales con el objetivo de inducir la tolerancia o prevenir el desarrollo de sensibilizaciones posteriores.

\section{Metodología}

La metodología fue de tipo descriptivo documental, que según señala Hernández, Fernández y Baptista (2010), está orientada a estudiar en forma intensiva las propiedades importantes de personas, grupos, comunidades o cualquier otro fenómeno sometido a análisis. Por otro lado, la investigación es de tipo documental que según Chávez (2007), implica un proceso de búsqueda, selección, lectura, registro, organización, descripción, análisis e interpretación de datos extraídos de fuentes documentales existentes en torno a un problema, con el fin de encontrar respuesta a los objetivos planteadas en cualquier área del conocimiento humano y desde esta perspectiva, el diseño fue no experimental. La población la constituyeron 120 madres que asistieron a la consulta durante el segundo semestre del año 2017. El instrumento utilizado fue un cuestionario estructurado de preguntas cerradas y 3 alternativas de repuestas, y el análisis de la información se hizo a través de la estadística descriptiva inferencial. El análisis de la información se procesó estadísticamente a través del paquete SPSS 23.0. 


\section{Resultados}

Se aplicó un instrumento tipo cuestionario a 120 madres que acudieron a la consulta externa con hijos en periodos de lactancia materna exclusiva al hospital Rodríguez Zambrano ubicado en el cantón de Manta de la Provincia de Manabí, Ecuador, en la que se obtuvieron los siguientes resultados:

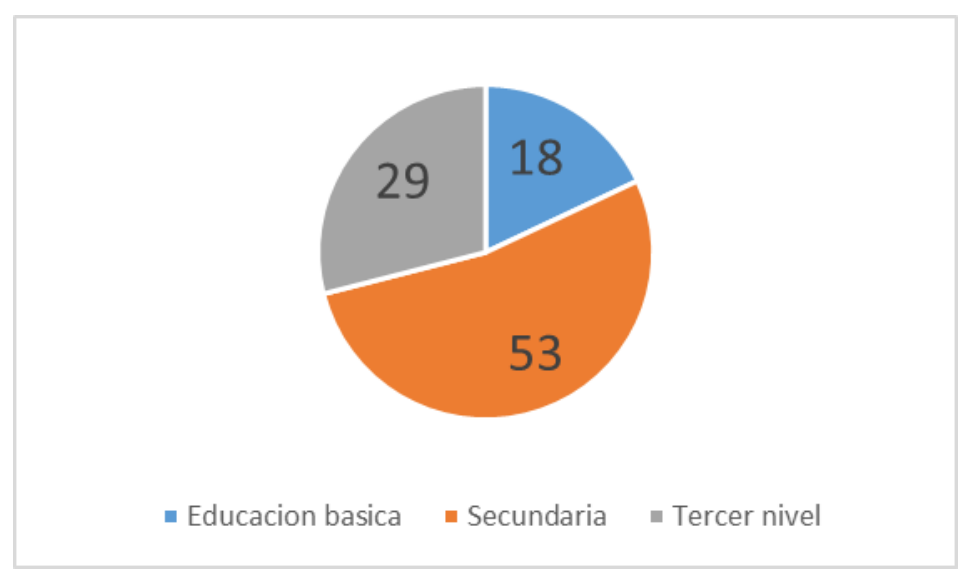

Figura 1: Nivel de escolaridad Materna

Fuente: Elaboración propia.

En cuanto al indicador nivel de escolaridad de las madres, se pudo constatar que el $18 \%$ de las habían completado la educación básica, el 53\% hasta culminar la secundaria y el resto con el 29\% tenían estudios de tercer nivel. Destacándose el hecho, en la cual el $67 \%$ se dedicaban a oficios del hogar y un $23 \%$ trabajaban fuera de su hogar.

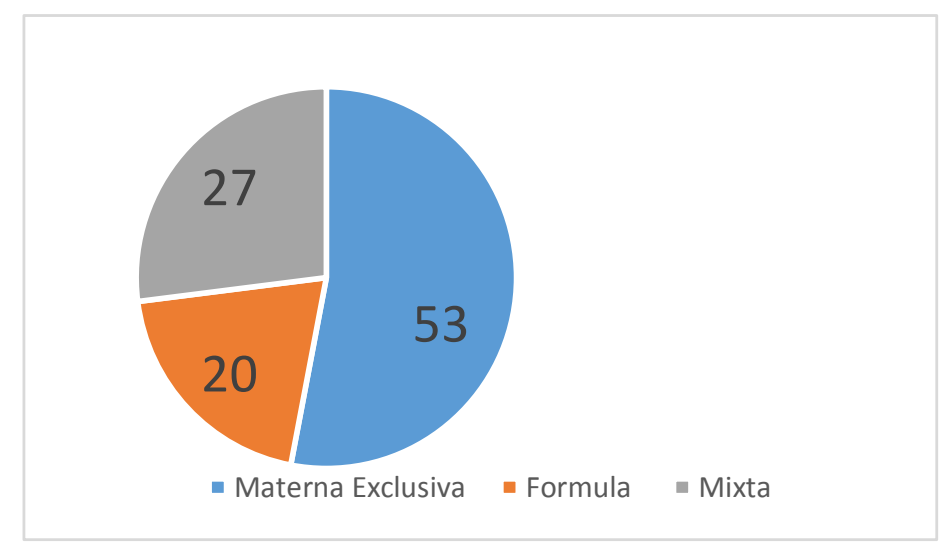

Figura 2: Inicio de alimentación

Fuente: Elaboración propia. 
El inicio de la alimentación en opinión del 53\% fue de tipo materna exclusiva, seguido del $20 \%$ que indico el inicio con formula sustitutiva de la lactancia materna y de forma combinada lo hizo el $27 \%$ de las encuestadas. Estos resultados, se complementaron con las siguientes razones de decisiones para con la alimentación del lactante, en primer término, las que suspendieron la lactancia fue motivado a la poca producción de leche materna, así como por terminar su licencia del trabajo y otras por circunstancias de complicaciones post parto que les impidió dar alimentación materna a su hijo.

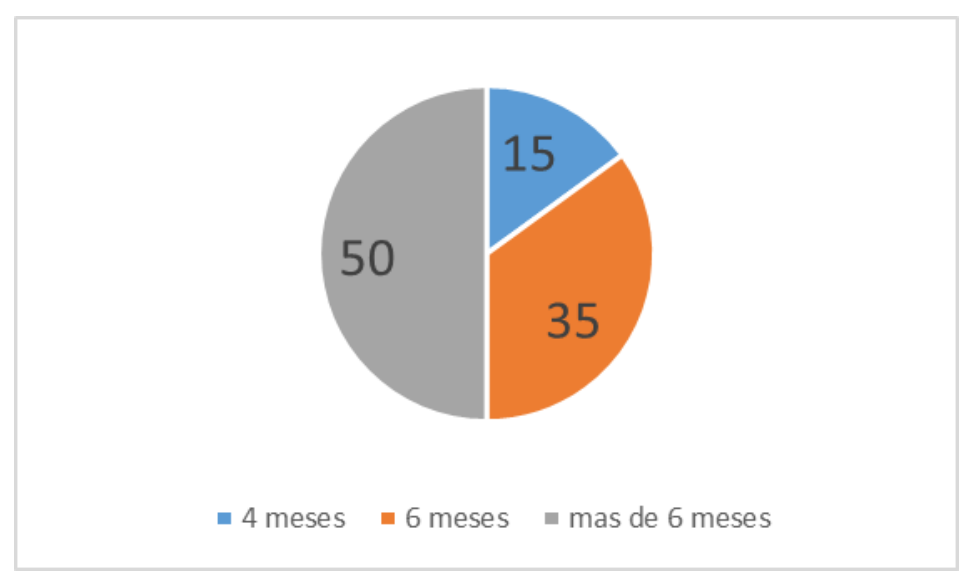

Figura 3: Inicio de alimentación Complementaria.

Fuente: Elaboración propia.

En opinión de las encuestadas, el inicio de la alimentación complementaria se inició a los 4 meses en el 15\% de los niños, el 35\% a los 5 meses y el 50\% indico después de los 6 meses de edad, e indicaron frutas, verduras, cereales y proteínas para el iniciaron la alimentación complementaria.

\section{Conclusiones}

Se determinó un descenso de la alimentación exclusiva de la leche materna en menores de 6 meses y un aumento de consumo de fórmulas y otras leches, indicando como causas de suspensión la poca producción de leche materna, por terminación de su licencia del trabajo y complicaciones post parto.

La alimentación complementaria, se dio considerando dentro del grupo de alimentos las frutas según la época de producción, verduras y cereales en mayor proporción, asimismo se utilizaron las proteínas cárnicas para complementar su alimentación.

Fue posible determinar, que entre las madres investigadas había poco conocimiento de cuáles son las ventajas de la alimentación exclusiva de leche materna al inicio de la alimentación del niño y 
el momento idóneo del inicio de la alimentación complementaria, decisión que tomaban sin consulta médica ni de especialista en la materia.

\section{Referencias Bibliográficas}

CEPAL. (2010). Salud materno-infantil de pueblos indígenas y afrodescendientes de América Latina: aportes para una relectura desde el derecho a la integridad cultural. Santiago de Chile: Naciones Unidas. Recuperado en: http://www.eclac.org/publicaciones/xml/9/41579/LCW.347Oct_2010.pdf Chávez (2007). Introducción a la Investigación Educativa. Maracaibo: Ars Gráfica. Hernández, Fernández y Baptista, (2010). Metodología de la Investigación. McGraw Hill. México.

OMS (2017). Organización Mundial de la Salud: Estrategia mundial para la alimentación del lactante y el niño pequeño. Organización Mundial de la Salud.

Torras, M (2001). Lactancia materna durante el embarazo y en tándem. En: Aguayo Maldonado J, eds. La lactancia materna. Sevilla: Universidad de Sevilla. Capítulo 10. Páginas 243-249.

UNICEF (1989). Protección, promoción y apoyo de la lactancia natural: la función especial de los servicios de maternidad. Ginebra: Organización Mundial de la Salud.

Vahlquist, B (1981). Organización Mundial de la Salud. Modalidades de la lactancia natural en la actualidad. Informe sobre el estudio en colaboración de la OMS acerca de la lactancia natural. Ginebra: Organización Mundial de la Salud

Wright, A (2001). Incremento de la lactancia materna en Estados Unidos. Pediatr Clin North Am (ed esp). 2001; 1:1-12. 
